\title{
Nefrectomía radical laparoscópica retroperitoneal: experiencia inicial con 50 casos
}

\author{
Silva Quintela R, Siqueira F, Marelli de Carvalho G, Miranda Salim M, Lopes Abelha Jr D, \\ Eduardo Távora J.
}

\begin{abstract}
Servicio de Urología. Hospital da Previdencia dos Servidores do Estado de Minas Gerais, Hospital Vila da Serra and Hospital da Baleia, Belo Horizonte, Minas Gerais.
\end{abstract}

Actas Urol Esp. 2008;32(4):417-423

\section{RESUMEN}

NEFRECTOMÍA RADICAL LAPAROSCÓPICA RETROPERITONEAL: EXPERIENCIA INICIAL CON 50 CASOS

Objetivo: La nefrectomía radical laparoscópica se realiza preferentemente mediante abordaje transperitoneal. A pesar de las ventajas que ofrece el abordaje laparoscópico retroperitoneal directo, no ha encontrado aceptación uniforme debido al pequeño espacio de trabajo en el retroperitoneo. La retroperitoneoscopia es nuestro abordaje preferido para realizar nefrectomía radical de tumores renales localizados. Presentamos nuestra técnica y nuestra experiencia con los primeros 50 casos de nefrectomía radical retroperitoneoscópica y comparamos los resultados con otras series.

Material y Métodos: Entre marzo de 2004 y marzo de 2007 se realizaron nefrectomía radical retroperitoneoscópica a 50 pacientes con tumores renales cT1/cT2. Se realizó nefrectomía con una técnica laparoscópica retroperitoneal con 4 puertos con el paciente colocado en posición de decúbito lateral. Se creó un balón artesanal para crear el espacio de trabajo retroperitoneal. La pieza quirúrgica se extrajo intacta por medio de una incisión ilíaca extraperitoneal. Los datos del seguimiento se revisaron retrospectivamente.

Resultados: El tamaño medio del tumor fue de 5,3 cm (3-13 cm), el tiempo quirúrgico fue de 150 min. (90-300 min), y la pérdida de sangre de $130 \mathrm{ml}$ (40-1000 ml). La estancia media en el hospital fue de 2,2 días (1-11 días). Hubo complicaciones en 6 (12\%) pacientes. Dos pacientes (4\%) tuvieron complicaciones importantes y uno de ellos necesito conversión a cirugía abierta. Cuatro pacientes (8\%) presentaron complicaciones menores. Hubo dos recidivas posteriores. Se produjeron una recidiva local en el trócar en un cáncer renal pT3aNOMO y una metástasis sistémica en un tumor urotelial pT3NOMO. Ambos casos presentaban extracción inadecuada de la pieza quirúrgica.

Conclusión: La retroperitoneoscopia es una alternativa viable, eficaz y segura para el tratamiento de tumores renales localizados. Se debe evitar la retroperitoneoscopia en tumores renales avanzados y de gran tamaño.

Palabras clave: Riñón. Laparoscopia. Nefrectomía. Cáncer renal. Retroperitoneoscopia.

\section{ABSTRACT}

RETROPERITONEAL LAPAROSCOPIC RADICAL NEPHRECTOMY: INICIAL EXPERIENCE WITH 50 CASES

Purpose: Laparoscopic radical nephrectomy is preferentially performed by transperitoneal approach. Despite offering advantages the direct retroperitoneal laparoscopic approach has not found uniform acceptance due to small working space in the retroperitoneum. Retroperitoneoscopy is our preferred approach for performing radical nephrectomy for localized renal tumors. We present our technique and our experience with the first 50 retroperitoneoscopic radical nephrectomies and compare the results with other series.

Matherials and Methods: 50 patients underwent retroperitoneoscopic radical nephrectomy for renal tumors cT1/cT2 between march 2004 to march 2007. A four ports retroperitoneal laparoscopic nephrectomy technique is performed with the patient in the full flank position. An artisanal balloon is used to create the retroperitoneal working space. The specimen is extracted intact by an extraperitoneal iliac incision. Follow up data were retrospectively reviewed.

Results: Mean tumor size was 5,3 cm ( 3 to $13 \mathrm{~cm}$ ), surgical time was $150 \mathrm{~min}$ (90 to $300 \mathrm{~min}$ ), and blood loss was $130 \mathrm{ml}$ (40-1000 ml). Average hospital stay was 2,2 days (1-11 days). Complications occurred in 6 (12\%) patients. Two patients (4\%) presented major complications and one of then require open conversion. Four patients (8\%) presented minor complications. There were two later recurrences. One local and port site recurrence in a pT3aNOMO renal cancer and one systemic metastases in a pT3NOMO urothelial cancer occurred. Both cases presented inadequately extraction.

Conclusion: Retroperitoneoscopy is a feasible, effective and safe alternative for the treatment of localized renal tumors. Retroperitoneoscopy should be avoided in advanced and large size renal tumors.

Keywords: Kidney, Laparoscopy, Nephrectomy, Renal cancer, Retroperitoneoscopy. 
$\mathrm{L}$ a nefrectomía radical laparoscópica para el tratamiento del cáncer renal está realizándose cada vez más en varios centros mundiales ${ }^{1-4}$. Se ha demostrado que tiene resultados similares a los de la cirugía abierta en el control del cáncer y en la supervivencia, con beneficios en cuanto a recuperación del paciente y morbilidad ${ }^{3-6}$. A pesar de que, por lo general, el abordaje retroperitoneal es el preferido en la nefrectomía radical abierta, el abordaje transperitoneal es la técnica favorita en los procedimientos laparoscópicos. La nefrectomía radical retroperitoneoscópica se ha descrito, pero se ha realizado de forma mas limitada $^{1,2}$. No ha encontrado aceptación debido a la pequeña área de trabajo y la percepción de dificultad al extraer especimenes más grandes. Sin embargo el abordaje retroperitoneoscópico ofrece acceso directo al pedículo renal y menor morbilidad debido a la preservación de la cavidad peritoneal. Hasta la fecha el abordaje retroperitoneoscópico es la técnica preferida para realizar la nefrectomía radical en algunos centros urológicos de excelencia laparoscópica ${ }^{1,2}$.

Presentamos nuestra experiencia inicial de nefrectomía radical retroperitoneoscópica con 50 pacientes. Describimos nuestra técnica quirúrgica y resultados que se comparan con otras series laparoscópicas.

\section{MATERIALES Y MÉTODOS}

De marzo 2004 a marzo 2007, realizamos 50 nefrectomías radical retroperitoneoscópicas a 50 pacientes con cáncer renal. Todos los casos fueron realizados por un único cirujano (RSQ). El tamaño medio del tumor fue de 5,3 cm (rango 2 a $13 \mathrm{~cm}$.), y en el CT preoperatorio, ningún paciente mostró evidencia extra renal, ganglios o extensión a la vena renal. Cuarenta y nueve pacientes presentaban masas renales sólidas y un paciente tenía un quiste renal complejo tipo Bosniak III. Los datos demográficos se presentan en la Tabla 1.

El tiempo quirúrgico se midió desde el momento de la incisión hasta el último punto. La pérdida de sangre se estimó basándose en el volumen del aspirador. Todas las piezas quirúrgicas fueron extirpadas intactas. El estadio patológico de los tumores se definió de acuerdo con la clasificación TNM y los datos del seguimiento se obtuvieron revisando los gráficos de los pacientes.

La tarde anterior a la cirugía se administraron $500 \mathrm{ml}$. de solución salina glicerinada para preparar
Tabla 1. Datos demográficos.

\begin{tabular}{lc}
\hline $\mathrm{N}^{\circ}$ de pacientes & 50 \\
Edad. Años (rango) & $59(30-83)$ \\
Lateralidad. Derecho/Izquierdo & $27 / 23$ \\
Género. Hombre/Mujer & $22 / 28$ \\
Tamaño. Centímetros (rango) & $5,5(3-13)$ \\
Estadio clínico (n) & \\
cT1a & 26 \\
cT1b & 14 \\
cT2 & 10 \\
\hline
\end{tabular}

el intestino. Inmediatamente antes de la anestesia se administraron por vía intravenosa antibióticos de amplio espectro. Después de la anestesia general y de la colocación de un catéter Foley se colocó al paciente en posición de decúbito sobre el lado no afectado. Se elevó el puente renal y la mesa se flexiona para ampliar la distancia costo-ilíaca.

Técnica quirúrgica: Se hace una incisión de 1,5 $\mathrm{cm}$, por debajo de la punta superior de la duodécima costilla. Se separan los músculos lumbares y se realiza una incisión en la fascia toracolumbar. Se realiza una disección roma con el dedo para desplazar anteriormente el peritoneo anteriormente. Se inserta un globo de Gaur (dedo de guante quirúrgico adjunto a un catéter de nelaton 16FR - (Fig. 1) posteriormente entre la fascia del psoas y la fascia de Gerota y se insufla con 500 a 800 cc, de solución salina para crear el espacio retroperitoneal. Se retira el globo y se fija un trocar Hasson de $10 \mathrm{~mm}$, a la

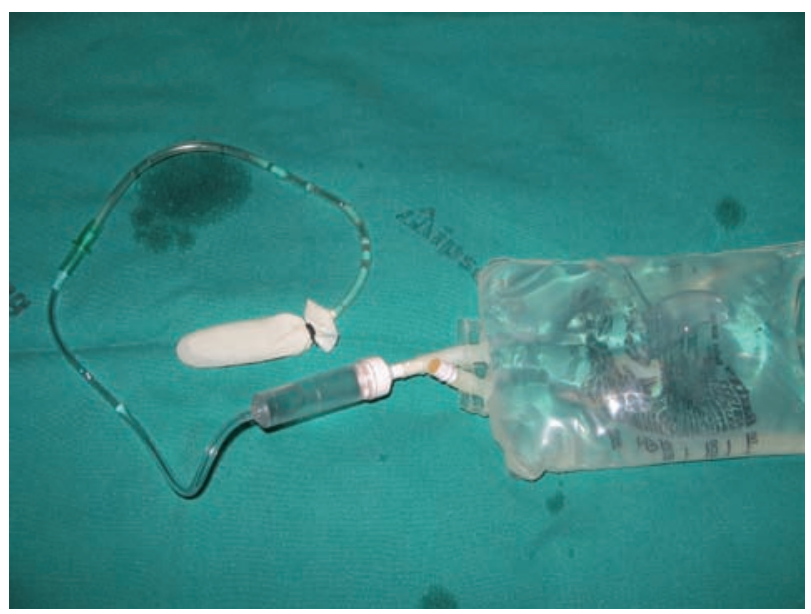

FIGURA 1. Balón de Gaur (hecho con una sonda tipo Nelaton $y$ un dedo de guante atado en su punta) conectado a un contenedor con solución salina para infusión y creación del espacio retroperineal. 
aponeurosis con puntos. Se establece un pneumoperitoneo de 12 a $15 \mathrm{~mm} \mathrm{Hg}$. Se colocan otros tres trocares (Fig. 2). La óptica de $0^{\circ}(10 \mathrm{~mm})$ se inserta en el primer trocar.

Las pinzas del asistente retraen el riñón anteriormente. Se identifican los puntos de referencia: el músculo psoas, el uréter la vena gonadal y la vena cava en el lado derecho. Se identifica el pedículo renal por las pulsaciones arteriales. Se diseca la arteria renal y se colocan clips metálicos de oclusión, con sección posterior. La vena renal se moviliza y controla con clips Hem-o-Lock® (Fig. 3). Se realiza una disección suprahilar a lo largo del borde medial del polo superior del riñón y la movilización

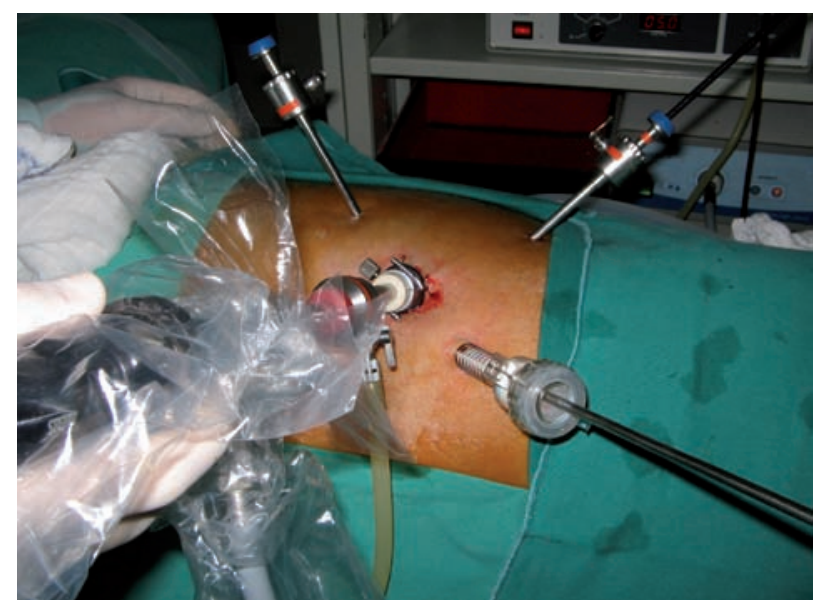

FIGURA 2. Vista superior de un paciente en posición decúbito lateral izquierdo para nefrectomía derecha con cuatro trocares. El trocar de Hasson en el borde de la duodécima costilla con la óptica, dos trocares de $5 \mathrm{~mm}$, en la línea axilar anterior y el trócar de $10 \mathrm{~mm}$, por encima de la cresta ilíaca.

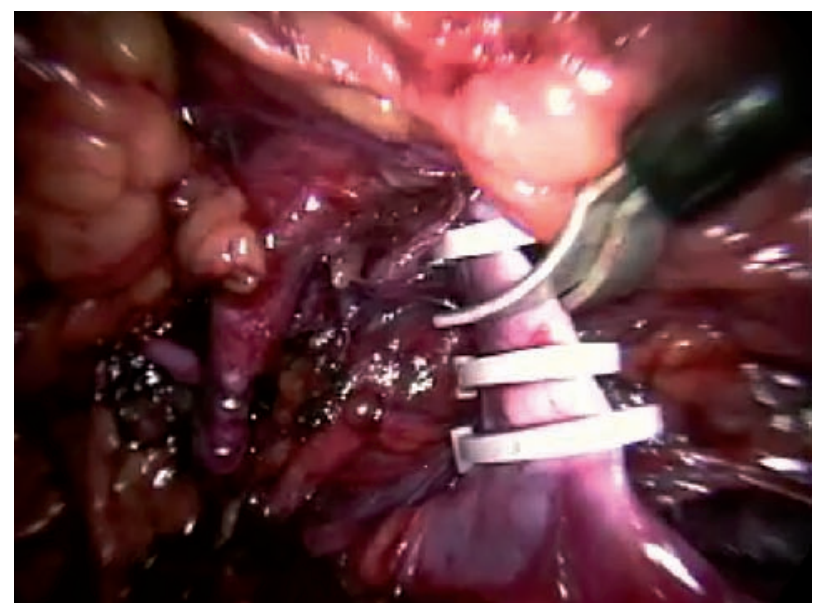

FIGURA 3. Pediculo renal derecho con la arteria renal controlada y seccionada con clips metálicos y la vena renal controlada por Hem-o-Lock®, siendo seccionada. en bloque de la glándula suprarrenal. La disección completa se realiza fuera de la fascia de Gerota. El uréter y la vena gonadal están sujetos y el espécimen está completamente liberado. La pieza quirúrgica se introduce en una endobolsa (Lapsac "-Cook Urological /Spencer, Indiana) y se extrae por una incisión iliaca extraperitoneal (Gibson).

\section{RESULTADOS}

Los datos intraoperatorios se detallan en la Tabla 2. El tiempo quirúrgico medio fue de $150 \mathrm{~min}$, (90-300 min.) y la pérdida media estimada de sangre de $130 \mathrm{ml}$ (50-1000 ml). No hubo transfusiones. El tamaño medio del tumor fue de $5,5 \mathrm{~cm}(3-13 \mathrm{~cm})$ $\mathrm{y}$ todos los especimenes fueron extirpados intactos en la endobolsa excepto en 5 pacientes que requirieron extracción manual. El promedio de longitud de la incisión para extirpar el espécimen fue de 6,5 $\mathrm{cm}$. La adrenalectomía en bloque se realizó en 40 (80\%) pacientes. De los 10 pacientes a los que no se les realizó adrenalectomía, 7 fueron preservadas de manera intencionada y los otros 3 debido a dificultades técnicas.

La estancia media hospitalaria fue de 2,2 días (rango 1 a 11 días). Hubo seis (12\%) complicaciones. Cuatro $(8 \%)$ complicaciones menores (3 pacientes con infección superficial del lugar del trocar y 1 pacientes con ileo prolongado) y 2 (4\%) con complicaciones graves (una lesión de la vena gonadal cerca de la vena cava en una nefrectomía radical derecha controlada después de conversión a cirugía abierta y una lesión en el colón izquierdo suturada mediante

Tabla 2. Datos intra y postoperatorios

\begin{tabular}{ll}
\hline $\begin{array}{l}\text { Media tiempo quirúrgico. } \\
\text { Minutos (rango) }\end{array}$ & 150 (90-300) \\
$\begin{array}{l}\text { Pérdida de sangre estimada. } \\
\text { Mililitros (rango) }\end{array}$ & 130 (50-1.000) \\
$\begin{array}{l}\text { Media longitud incisión. } \\
\text { Centímetros (rango) }\end{array}$ & $6,5(3-10)$ \\
$\begin{array}{l}\text { Media estancia hospitalaria. } \\
\text { Días (rango) }\end{array}$ & $2,2(1-11)$ \\
Conversión a cirugía abierta, $\mathrm{n}^{\circ}(\%)$ & $1(2)$ \\
Complicaciones menores, $\mathrm{n}^{\circ}(\%)$ & $4(8)$ \\
Complicaciones graves, $\mathrm{n}^{\circ}(\%)$ & $2(4)$ \\
Media seguimiento. Meses (rango) & $11(1-37)$
\end{tabular}


abordaje laparoscópico que presentó una fístula colónica extraperitoneal que cerró de forma espontánea tras 10 días de dieta pobre en residuos). No hubo peritoneotomía intencional, sin embargo se produjeron casualmente aperturas del peritoneo que no causaron problemas técnicos. Sólo fue necesaria una conversión a nefrectomía abierta según se ha mencionado previamente.

El estudio anatomo-patológico confirmó que 45 (90\%) de las piezas correspondian a carcinoma de células renales, $1(2 \%)$ carcinoma de células transicionales (CCT), 2 (4\%) angiomiolipomas, 1 (2\%) oncocitoma y 1 (2\%) quiste hemorrágico. Los estadios patológicos finales de los 46 pacientes con cáncer renal fueron 36 (72\%) pT1NOMO, 7 (14\%) pT2NOMO y 3 (6\%) pT3NOMO.

El promedio de seguimiento fue de 11 meses (rango 1 a 37 meses). Dos pacientes fallecieron al octavo y al vigésimo segundo meses respectivamente a causa de enfermedad metastásica. El primero presentaba CCT pT3NOMO y enfermedad metastásica diseminada. El uréter no se extirpó en el momento de la nefrectomía sino dos meses más tarde. El otro presentaba un cáncer de células renales de 13 cm., pT3aNOMO y desarrolló recidiva en el sitio de entrada del trocar 18 meses después de la cirugía (Fig. 4). En estos pacientes, la extracción de la pieza se realizó sin inclusión en la endobolsa. La supervivencia libre de enfermedad fue del 96\%, así como la supervivencia total.

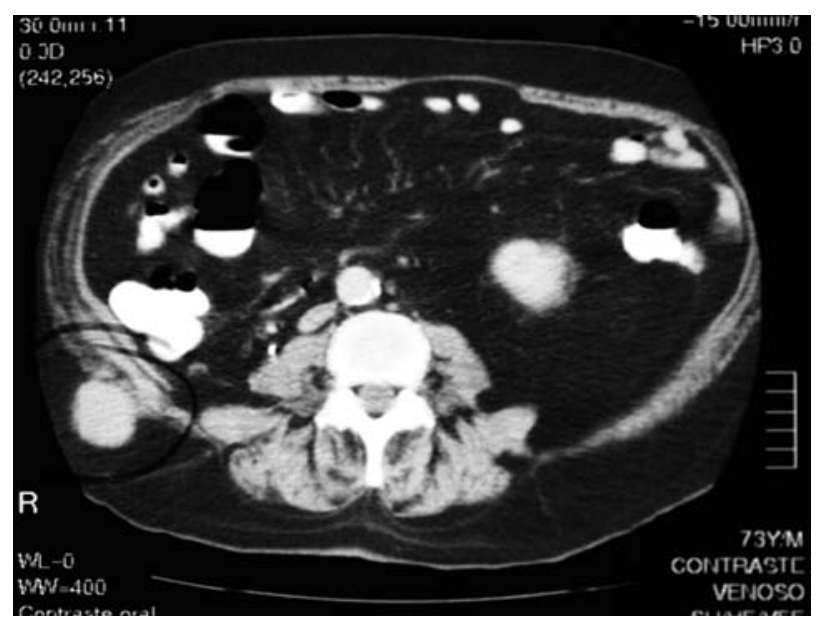

FIGURA 4. Tomografia computarizada (TC) mostrando metástasis en la localización del trócar en un varón de 65 años de edad después de una nefrectomia radical retroperitoneoscopia por cáncer de células renales de 13 cm, pT3aNOMO. La pieza quirúrgica se extirpó sin incluirlo en una endobolsa.

\section{DISCUSIÓN}

El abordaje laparoscópico se ha convertido en el acceso preferencial para la realización de nefrectomía radical en tumores renales localizados que no son subsidiarios de cirugía conservadora renal en varios centros mundiales ${ }^{1-6}$. La reducción de la morbilidad, rápida recuperación post-quirúrgica, y un mejor resultado cosmético de la laparoscopia son algunas de las ventajas de este abordaje si se le compara con el método tradicional abierto. Aunque las ventajas de la laparoscopia en relación a la morbilidad están bien definidas, todavía existen algunas controversias en relación con su control oncológico. La nefrectomía radical abierta (NRA) presenta una tasa de recidiva local de 2,5 a $5,5 \%$, para tumores iguales o mayores a pT2, y el tiempo medio de recurrencia es de 20 meses $^{7,8}$. En relación con el abordaje laparoscópico, los primeros trabajos sugieren que su eficacia oncológica tiene aún un corto seguimiento (hasta 36 meses) y un pequeño número de pacientes $^{3,4}$. En un estudio multicéntrico, Portis et al, compararon los grupos sometidos a NRA frente a los de NRL, en un total de 133 pacientes, con un seguimiento de 94 meses, obteniendo una tasa de supervivencia libre de enfermedad a 5 años de $91 \%$ y $92 \%$, respectivamente $(\mathrm{p}=0,58)$ y supervivencia cáncer específica de $92 \%$ y $98 \%$, respectivamente $(\mathrm{p}=0,12)^{9}$. Estos resultados fueron posteriormente reproducidos por Cadeddu et al y Saika et $\mathrm{al}^{5,6}$.

Recientemente, Permkongkosol et al demostraron la equivalencia oncológica entre la NRL y la NRA en 10 años de seguimiento ${ }^{10}$. A pesar del corto seguimiento nuestra serie, presenta una supervivencia libre de enfermedad y supervivencia global, compatible con lo reflejado en la literatura. Los dos casos de recidiva se produjeron en tumores localmente avanzados (pT3). Se produjo un caso de recidiva sistémica en un tumor de células transicionales que invadía el parénquima renal, cuyo diagnóstico se realizó sólo con el análisis histológico y la resección ureteral no fue completa. El otro caso de recidiva local con implantes en el lugar del trocar se produjo en un hombre de 65 años que presentaba una gran tumor renal pT3a cuya extracción no se realizó correctamente; se hizo directamente a través de la incisión, sin utilizar la bolsa de protección.

La recidiva en el lugar de entrada del trocar tras NRL Y NRLR se puede consultar en la literatura ${ }^{1-13}$. Existe controversia en cuanto a si esta situación 
está relacionada con el abordaje laparoscópico o con el comportamiento tumoral. La mayoría de los casos publicados se produjeron en tumores agresivos y avanzados con los que hubo alguna clase de problema técnico durante el procedimiento o en el momento de la extracción de la pieza, como ocurrió en el caso descrito. Nuestra experiencia demuestra que, en tumores de gran tamaño y avanzados la probabilidad de problemas técnicos aumenta debido a la limitación de la zona de trabajo en la retroperitoneoscopia por lo que en estos casos es preferible utilizar otros abordajes.

El abordaje retroperitoneoscópico proporciona acceso directo al hilio renal que se realiza de manera posterior, sin necesidad de movilizar previamente las estructuras intraperitoneales. En la cirugía oncológica, este acceso permite el control precoz arterial, que se suele situar en relación posterior a la vena y con una mínima movilización renal. La no apertura de la cavidad peritoneal favorece la reducción de la causa de morbilidad y se reduce el íleo postoperatorio y el riesgo de lesión de órganos intraperitoneales. En algunos de nuestros pacientes se produjo una apertura ocasional e involuntaria del peritoneo durante la disección o la creación del espacio. Aunque este hecho no tiene una importante repercusión durante la cirugía, se observó una diferencia en relación con el malestar abdominal y en el regreso del peristaltismo, que fueron más lentos en pacientes en los que el $\mathrm{CO}_{2}$ entró en el peritoneo.

El reto del abordaje retroperitoneal es trabajar en un espacio tan pequeño. La cirugía laparoscópica en la zona retroperitoneal requiere una orientación más precisa, un movimiento más delicado, y un equipo mejor coordinado en el abordaje transperitoneal. Gill et al informaron que el tamaño de la masa renal no es un problema importante para la nefrectomía retroperitoneal si se crea un adecuado espacio retroperitoneal, y recomendaron que se realice con una adecuada dilatación de globo ${ }^{2}$. El embolsado y extracción de las piezas quirúrgicas es el paso más difícil de realizar en espacios pequeños. La limitación de la zona de trabajo y la incompatibilidad entre el diámetro de la pieza y la bolsa fueron los factores que nos llevaron a realizar la extirpación de los especimenes sin bolsa de protección en 5 pacientes de esta serie. En los casos de grandes tumores esta limitación se debe tener en cuenta al escoger el abordaje retroperitoneoscópico. Sin embargo, la lite- ratura demuestra que, con más experiencia, es posible realizar una nefrectomía laparoscópica retroperitoneal segura incluso en casos complejos $2,14,15$.

A pesar de que ofrece ventajas, el abordaje retroperitoneal está mucho menos extendido que el transperitoneal para la nefrectomía radical. En la literatura existen pocos trabajos que presenten una comparación de los dos abordajes. En un trabajo prospectivo y aleatorio, Desai et al valoraron 102 pacientes sometidos a transperitoneal (50 pacientes) y retroperitoneal (52 pacientes) NRL, y demostraron que los dos abordajes son comparables en relación con hemorragia, complicaciones, analgesia post-quirúrgica, tiempo de hospitalización y control oncológico ${ }^{16}$. Sin embargo, el abordaje retroperitoneal muestra un tiempo quirúrgico menor. Estos resultados son comparables a otros estudios prospectivos y aleatorios ${ }^{17}$.

Las complicaciones ocurrieron en 6 (12\%) pacientes de nuestra serie. Nuestra tasa de complicaciones está en concordancia con otras series ${ }^{1,2,18}$. Analizando específicamente las complicaciones durante el retroperitoneoscopia, Meraney et al informaron una incidencia de lesiones vasculares y viscerales del 1,7 y 0,25\%, respectivamente, en 404 procedimientos de varias patologías renales y suprarrenales ${ }^{18}$. Estos resultados son comparables a las series de nefrectomía transperitoneal ${ }^{19}$. En esta serie, nosotros sólo tuvimos una complicación vascular que necesitó conversión. Era una mujer de 63 años que tenía un tumor del polo inferior renal, y, durante la movilización de la grasa de Gerota se produjo una lesión de la vena gonadal derecha, en su inserción en la vena cava. La posición en la mesa quirúrgica es también una ventaja del abordaje retroperitoneoscópico, ya que hace más fácil convertirlo a acceso anatómico de lumbotomía.

Otra complicación importante de esta serie está representada por una lesión extraperitoneal del colon izquierdo. Las lesiones de órganos intraperitoneales pueden ocurrir aun con el abordaje extraperitoneal, como se observa en este caso de una paciente que presentaba un quiste renal complejo adherido al colon descendente que resultó ser un quiste hemorrágico en el análisis histológico. Se abrió el colon y la lesión fue diagnosticada y suturada durante la cirugía, pero tres días después se manifestó una fístula colónica que permitió un tratamiento conservador sin necesidad de reintervención ya que estaba aislada del peritoneo. 
En esta serie, la morbilidad relacionada con la herida representa una complicación menor. Como se observó en tres pacientes de este grupo la retroperitoneoscopia, que se desarrolla en un área anatómica con músculos más gruesos, como la región lumbodorsal, presenta un mayor riesgo de traumatismos musculares en los lugares de entrada de los trocares, por lo general el primer trocar, que favorece la formación de equimosis, seromas y abscesos de pared.

Nuestros resultados muestran que el abordaje retroperitoneal es eficaz y seguro para la NRL. La elección del abordaje debe basarse en la experiencia del cirujano y la preferencia. Sin embargo, en algunas situaciones, el abordaje retroperitoneal puede presentar ventajas, como en el caso de los pacientes con cirugía abdominal previa o pacientes obesos ${ }^{14}$. Por otro lado, este abordaje debería evitarse en pacientes con lumbotomía previa y en los pacientes con tumores grandes y avanzados (mayor de $7 \mathrm{~cm}$ de diámetro, asociado con trombo venoso, con adenopatías o invasión de estructuras adyacentes) ${ }^{15}$.

Aunque el abordaje transperitoneal es el más utilizado en la nefrectomía radical laparoscópica, creemos que el abordaje retroperitoneal presenta ventajas y debe formar parte del arsenal terapéutico del cirujano urológico.

\section{CONCLUSIÓN}

La nefrectomía radical retroperitoneoscopia es una alternativa viable, eficaz y segura para el tratamiento de tumores renales localizados. El acceso laparoscópico retroperitoneal ofrece acceso directo al pedículo renal y produce menos íleo postoperatorio debido a la preservación de la cavidad peritoneal. El abordaje retroperitoneal laparoscópico de la nefrectomía radical debe evitarse en tumores renales de gran tamaño si se percibe dificultad para extracción de la pieza quirúrgicas.

\section{REFERENCIAS}

1. Abbou CC, Cicco A, Gasman D, Hoznek A, Antiphon P, Chopin $\mathrm{Dk}$, et al. Retroperitoneal laparoscopic versus open radical nephrectomy. J Urol. 1999;161(6):1776-1780.

2. Gill IS, Schweizer D, Hobart MG, Sung GT, Klein EA, Novick AC. Retroperitoneal Laparoscopic radical nephrectomy: the Cleveland Clinic experience. J Urol. 2000;163(6):1665-1670.

3. Ono Y, Kinukawa T, Hattori R, Yamada S, Nishiyama N, Mizutani $\mathrm{K}$, et al. Laparoscopic radical nephrectomy for renal cell carcinoma: a five year experience. Urology. 1999;53(2):280-285.

4. Dunn MD, Portis AJ, Shalhav AL, Elbahnasy AM, Heidron C, McDougall EM, et al.: Laparoscopic versus open radical nephrectomy: a 9-year experience. J Urol. 2000;164(4):1153-1157.
5. Cadeddu JA, Ono Y, Clayman RV, Barrett PH, Janetschek G, Fentie DD, et al: Laparoscopic radical nephrectomy for renal cancer: evaluation of efficacy and safety: a multicentric experience. Urology. 1998;52(5):773-777.

6. Saika T, Ono Y, Hattori R, Gotoh M, Kamihira O, Yoshikawa Y, et al. Long-term outcome of laparoscopic radical nephrectomy for pathologic T1 renal cell carcinoma. Urology. 2003;62(6): 1018-23.

7. Giuliani L, Giberti C, Martorana G, Rovida S. Radical Extensive Surgery for renal cell carcinoma: long term results and prognostic factors. J Urol. 1990;143(3):468-472.

8. Pantuck AJ, Zisman A, Dorey F, Chão DH, Han KR, Said J, et al. Renal cell carcinoma with retroperitoneal limphonodes. Role of limphonode dissection. J Urol. 2003;169(6):2076-80.

9. Portis AJ, Yan Y, Landman J, Chen C, Barret PH, Fentie DD, et al. Long-term followup after laparoscopic radical nephrectomy. J Urol. 2002;167(3):1257-1260.

10. Permpongkosol S, Chan DY, Link RE, Sroka M, Allaf M, Varkarakis $\mathrm{I}$, et al. Long-term survival analisys after laparoscopic radical nephrectomy. J Urol. 2005;174(4 Pt 1):1222-1227.

11. Dhobada S, Patankar S, Gorde V. Case report: port-site metastasis after laparoscopic radical nephrectomy for renal-cell carcinoma. J Endourol. 2006;20(2):119-122.

12. Rassweiler J, Tsivian A, Kumar AV, Lymberakis C, Schulze M, Seeman O, Frede T. Oncological safety of laparoscopic surgery for urological malignancy: experience with more than 1,000 operations. J Urol. 2003;169(6):2072-2075.

13. Hetet JF, Rigaud J, Muller B, Leaute F, Renaudin K, Bochot O, et al. Unusual metastatic spread after converted laparoscopic radical nephrectomy: natural history of the tumour or role of laparoscopy?. Prog Urol. 2005;15(4):718-721.

14. Berglund RK, Gill IS, Babineau D, Desai M, Kaouk JH. A prospective comparison of transperitoneal and retroperitoneal laparoscopic nephrectomy in the extremely obese patient. BJU Int. 2007;99(4):871-874.

15. Steinberg AP, Finelli A, Desai MM, Abreu SC, Ramani AP, Spalviero M, et al. Laparoscopic radical nephrectomy for large (greater than $7 \mathrm{~cm}$, T2) renal tumors. J Urol. 2004;172(6 Pt 1): 2172-2176.

16. Desai MM, Strzemokowski B, Matin SF, Steinberg AP, Meraney $\mathrm{AM}$, Kaouk JH, et al. Prospective randomize comparison of transperitoneal versus retroperitoneal laparoscopic radical nephrectomy. J Urol. 2005;173(1):38-41

17. Nambirajan T, Jeschke S, Al-Zahrani H, Vrabec G, Leeb K, Janetschek G. Prospective, randomized controlled study: transperitoneal laparoscopic versus retroperitoneoscopic radical nephrectomy. Urology. 2004;64(5):919-24.

18. Meraney AM, Samee AA, Gill IS. Vascular and bowel complications during retroperitoneal laparoscopic surgery. J Urol. 2002; 168(5): 1941-1944.

19. Fahlenkamp D, Rassweiler J, Fornara P, Frede T, Loening SA. Complications of laparoscopic procedures in urology: experience with 2,407 procedures at Germans centers. J Urol. 1999; 162(3 Pt 1):765-770.

20. Gaur DD; Agarwal DK; Purohit KC: Retroperitoneal Laparoscopic nephrectomy: initial case report. J Urol. 1993;149(1): 103-105.

21. Robson CJ. Radical Nephrectomy for renal cell carcinoma. J Urol. 1963;89:37-42.

22. Clayman RV, Kavoussi LR, Soper NJ, Dierks SM, Meretyk S, Darcy MD, et al. Retroperitonial laparoscopic nephrectomy: Initial case report. J Urol. 1991 Aug; 146(2):278-282.

23. Kerbl K, Figenshau RS, Clayman RV, Chandhoke PS, Kavoussi LR, Albala DM, et al. Retroperitonial laparoscopic nephrectomiy: laboratory and clinical experience. J Endourol. 1993;7(1): 23-26. 
24. Clavien PA, Sanabria JR, Strasberg SM. Proposed classification of complications of surgery with examples of utility in cholecystectomy. Surgery. 1992 May;111(5):518-526.

25. Gill, IS; Meraney, AM; Schweiaer, DK; Savage, SS; Hobart, MG; Sung, GT et al. Laparoscopic radical nephrectomy in 100 patients: a single center experience from The United States. Cancer. 2001 Oct 1;92(7):1843-1855.

26. Barrett PH, Fentie DD, Taranger LA. Laparoscopic radical nephrectomy with morcellaton for renal cell carcinoma: The Saskatoon experience. Urology. 1998;52(1):23-28.

27. Richard, F; Schaetz, A; Chatelain, C. Facteurs pronostiques du cancer du rein. In: Seminares d`Uro-Nephrologie. Edit par C Chatelein et $\mathrm{Cl}$ Jacobs. Paris: Masson, chapt 14, pp157-175 1998.

28. Sung GT, Gill IS Anatomic landmarks and time management during retroperitonioscopic radical nephrectomy. J Endourol. 2002;16(3): 165-169.

29. Thiel R, Adams JB, Schulam PG, Moore RG, Kavoussi LR Venous dissection injuries during laparoscopic urological surgery. J Urol. 1996 Jun;155(6):1874-1876.
30. Rassweiler JJ, Seemann O, Frede T, Henkel TO, Alken P. Retroperitonioscopy: experience with 200 cases. J Urol. 1998; 160(4): 1265-1269

31. Tisdale BE, Kapoor A, Hussain A, Piercey K, Whelan JP. Intact specimen extraction in laparoscopic nephrectomy procedures: Pfannenstiel versus expanded port site incisions. Urology. 2007 Feb;69(2):241-244.

32. Meraney AM, Gill IS. Financial analysis of open versus laparoscopic radical nephrectomy and nephroureterctomy. J Urol. 2002;167(4):1757-1762.

33. Nadler RB, Loeb S, Clemens JQ, Batler RA, Gonzalez CM, Vardi IY. A prospective study of laparoscopic radical nephrectomy for T1 tumors-is transperitoneal, retroperitoneal or hand assisted the best approach?. J Urol. 2006;175(4):1230-1233; discussion 1234.

Correspondencia autor: Dr. R. Silva Quintela

Servicio de Urología

Hospital da Previdência dos Servidores do Estado de Minas Gerais

E-mail autor: quintelarod@yahoo.com

Información artículo: Original - Laparoscopia

Trabajo recibido: enero 2008

Trabajo aceptado: febrero 2008 